Results Totally 3,130 cases were recruited into the study. $54.6 \%$ were males, the first case had been reported in 1990, and the highest incident case had been reported in the year 2004 with 461 cases followed by 2005 (343), and 2006(302) respectively. The highest cumulative case had been reported from Mae Fah Luang Hospital (25.8\%), followed by Mae Suai hospital (18.8\%). 46.0\%were Akha, 19.7\%were Lahu, and $9.5 \%$ were Yao. 38.8\%were $31-40$ years old, followed by 21-30 years old(33.6\%), and $41-50$ years old (13.4\%). $44.4 \%$ were agriculture, $32.0 \%$ were employee. $91.6 \%$ were infected by sexual intercourse, $5.7 \%$ were mother to Child. $24.0 \%$ were receiving ARV, $30.7 \%$ were receiving OI treatment, and $9.5 \%$ were tested CD4 level. Male had higher of survival rate than female ( $p$-value $>0.001$ ), and male were younger than female at the age of infection ( $p$-value $>0.001$ ). There was statistically significant difference of mode of infection by tribe ( $p$-value $>0.001$ ).

Conclusion Specific health education programmes and empower them for using condom are needed to setting up for HIV/AIDS prevention and control among hill tribe people in Thailand.

\section{P3.220 INCIDENCE AND PREVALENCE OF HIV INFECTIONS AMONG FISHERMEN AROUND LAKE VICTORIA IN KISUMU KENYA}

doi:10.1136/sextrans-2013-051184.0677

${ }^{1,2,3} \mathbf{R} 0$ Ondondo, ${ }^{3} \mathrm{Z}$ W Ng'ang'a, 'S Mpoke, ' M K Kiptoo, 1,2,3 E A Bukusi. ' ${ }^{\prime}$ Kenya Medical Research Institute, Nairobi, Kenya; ${ }^{2}$ n Collaboration with University of California San Francisco, San Francisco, CA, United States; ${ }^{3}$ Jomo Kenyatta University of Agriculture and Technology, Nairobi, Kenya

Background Men with high-risk sexually behaviour are important drivers of HIV/STI infections in the general population. Prevention of HIV/STIs among such men could potentially reduce these infections especially among women - who bear the greatest HIV/STIs disease burden in sub Saharan Africa. We sought to understand immunovirology of HPV infections among fishermen. This evaluation highlights the rate of HIV infections in this population.

Methods Three hundred fishermen were recruited and followed up every 3 months for 1 year. HIV, syphilis serology, CD4/CD8 and complete blood count were evaluated and a demographic questionnaire administered. Data was analysed by SPSS ver18.

Results The 300 men recruited into this study had mean age of 28 years, $76 \%$ were married and $57 \%$ had only basic education. The mean for age of sexual debut and lifetime sexual partners was 15 years and 10 respectively. They had a modal sexual activity of 3 times/week and a mean of 3 rounds/sexual act. Sixty one (20\%) had practised oral sex. Thirty six (12\%) and $80(27 \%)$ of men washed their genitals, before and after sex respectively. A significant number of men; 183 (61\%) P < 0.01 and 118 (39\%) P < 0.05, never used condoms with their regular sexual partners and new sexual partners respectively. They had a mean CD4 and white blood cell (WBC) count of 830 cells $/ \mathrm{ml}$ and $5.6 \times 10^{9} / \mathrm{L}$ of blood. Baseline HIV and syphilis prevalence was $23 \%$ and $9 \%$ respectively. The HIV incidence during the 1 year follow-up was $4.2 \%$. Fishermen HIV burden was over 3 times the Kenyan national HIV prevalence of $7 \%$.

Conclusion Fishermen in this context, comprise a young sexually high-risk, highly-migratory population with high HIV incidence and prevalence. Low condom use coupled with concurrent multiple sexual partnerships make them a potential key population who require targeted prevention strategies to reduce HIV/STI infections and transmission.

\section{P3.221 ORAL AND INJECTABLE HORMONAL CONTRACEPTION DECREASE RISK OF BACTERIAL VAGINOSIS BUT ORAL CONTRACEPTION MAY INCREASE RISK OF VAGINAL CANDIDIASIS: A SYSTEMATIC REVIEW OF PUBLISHED AND UNPUBLISHED DATA}

doi:10.1136/sextrans-2013-051184.0678
J H van de Wijgert, ${ }^{2} \mathrm{M}$ C Verwijs, ${ }^{3} \mathrm{~A}$ Norris Turner, ${ }^{4} \mathrm{C}$ S Morrison. ${ }^{1}$ University of Liverpool, Institute of Infection and Global Health, Liverpool, UK; ${ }^{2}$ Amsterdam Institute of Global Health and Development (AIGHD), Amsterdam, The Netherlands; ${ }^{3} T$ The Ohio State University, Division of Infectious Diseases, Columbus, OH, United States: ${ }^{4} \mathrm{FH} 360$ Clinical Sciences, Durham, NC, United States

Background A recent World Health Organization (WHO) technical consultation concluded that combined oral contraception (COC) does not increase HIV acquisition in women, but the evidence for depot medroxyprogesterone acetate (DMPA) is conflicting. Significant evidence suggests that bacterial vaginosis (BV) and vaginal candidiasis, both representing an 'unhealthy' vaginal microbiome, increase HIV acquisition in women.

Methods We conducted a systematic review using the PRISMA 2009 guidelines, and re-analysed the Hormonal Contraception and HIV Acquisition (HC-HIV) study, to evaluate the effect of HC use on the vaginal microbiome. Vaginal microbiome outcomes included BV by Nugent scoring, vaginal candidiasis by culture or $\mathrm{KOH}$ wet mount, and microbiome compositions as characterised by molecular techniques.

Results Our review of 36 eligible studies found that COC and DMPA use reduce BV by $10-20 \%$ and $18-30 \%$, respectively. The $\mathrm{HC}-\mathrm{HIV}$ data showed that COC and DMPA use also reduce intermediate microbiota (Nugent score of $4-6$ ) by $11 \%$ for each. In contrast, COC use (but not DMPA use) may increase vaginal candidiasis; 7 of 12 studies reported a statistically significant increase in vaginal candidiasis, 2 reported a positive association approaching significance, 2 reported no association, and one reported a statistically significant reduction. Evidence for a reduction of BV risk in $\mathrm{HC}$ users is much stronger than evidence for a potential increased candidiasis risk in COC users: the quality of the BV studies was higher and the results more consistent. Molecular vaginal microbiome studies $(n=4)$ confirm that high oestrogen levels favour a vaginal microbiome composition dominated by 'healthy' Lactobacillus species; the effects of progesterone on the microbiome are less clear.

Conclusions The hypothesis that DMPA use may increase HIV risk by increasing BV or vaginal candidiasis risk is not supported by the evidence. COC use may predispose for vaginal candidiasis, but is not believed to be associated with increased HIV acquisition

\section{P3.222 HIGH RISK HUMAN PAPILLOMAVIRUS VIRAL LOAD AND PERSISTENCE AMONG HIV-NEGATIVE AND HIV-POSITIVE MEN}

doi:10.1136/sextrans-2013-051184.0679

${ }^{1} \mathbf{M}$ K Grabowski, ${ }^{2,1} \mathrm{R}$ H Gray, ${ }^{2,3} \mathrm{D}$ Serwadda, ${ }^{4} \mathrm{G}$ Kigozi, ${ }^{1,5} \mathrm{P}$ E Gravitt, ${ }^{2} \mathrm{~F}$ Nalugoda, ${ }^{1,2,6} \mathrm{~S}$ J Renyolds, ${ }^{1,2} \mathrm{M}$ Wawer, ${ }^{1,6} \mathrm{~T}$ C Quinn, ${ }^{1,7} \mathrm{~A}$ A R Tobian. ' ${ }^{1}$ Johns Hopkins, Baltimore, MD, United States; ${ }^{2}$ Rakai Health Sciences Program, Entebbe, Uganda; ${ }^{3}$ School of Public Health, Makerere University, Kampala, Uganda; ${ }^{4}$ Rakai Health Sciences Program, En, Uganda; ${ }^{5}$ Perdana University Graduate School of Medicine, Serdang, Malaysia; ${ }^{6}$ Division of Intramural Research, National Institute of Allergy and Infectious Diseases, National Institutes of Health, Bethesda, MD, United States; 'Rakai Health Sciences Program, Entebbe, Austria

Background High-risk human papillomavirus (HR-HPV) viral load is associated with transmission and persistence in women. It is unknown whether viral load is associated with HR-HPV persistence in HIV-negative or HIV-positive men.

Methods 703 HIV-negative and 233 HIV-positive heterosexual men participated in a male circumcision trial in Rakai, Uganda. Penile swabs were tested at enrollment and 6,12 and 24 months for HR-HPV using the Roche HPV Linear Array, which provides a semiquantitative measure of HPV shedding by hybridization band intensity (graded:1-4). Prevalence risk ratios (PRR) were used to estimate the association between HR-HPV viral load and persistent detection of type-specific HR-HPV infection. 
Results At least one HR-HPV was identified in 264 HIV-negative men (37.6\%, 403 genotypes total) and 164 HIV-positive men $(70.4 \%$, 399 genotypes total) at enrollment. Among HIV-negative men, younger and unmarried men were more likely to have higher viral loads. HR-HPV genotypes with high viral load (grade:3-4) at enrollment were more likely to persist than HR-HPV genotypes with low viral load (grade:1-2) among HIV-negative (month 6: adjPRR = 1.80, 95\% CI: 1.31-2.47; month 12: adjPRR $=2.04$, 95\% CI: 1.39-3.01), and HIV-positive men (month 6: adjPRR = 1.33, 95\% CI: 1.06-1.67; month 12: adjPRR $=1.70,95 \% \mathrm{CI}: 1.16-2.50)$. Long-term persistence of HR-HPV was more frequent among HIV-positive men compared to HIV-negative men (month 24: adjPRR $=2.24,95 \% \mathrm{CI}$ : 1.46-3.45), and HR-HPV infections with low viral loads were detected more frequently among HIV-positive men at all follow-up visits (6 months: PRR $=1.81,95 \%$ CI: 1.17-2.97; 12 months: PRR $=1.43$, 95\% CI: $0.8-2.4 ; 24$ months: PRR $=2.9$, 95\% CI: $1.53-$ 5.53)

Conclusions HR-HPV genotypes with high viral load are more likely to persist among HIV-negative and HIV-positive men, though persistence was more common among HIV-positive men. The results may explain the association between high HR-HPV viral load and transmission to women and increased levels of HR-HPV persistence in HIV-positive men.

\section{P3.223 HPV GENOTYPE DISTRIBUTION IN HIV-POSITIVE AFRICAN WOMEN AND ASSOCIATIONS WITH HIGH GRADE HISTOLOGICAL LESIONS BY CD4+ COUNT}

doi:10.1136/sextrans-2013-051184.0680

${ }^{1} \mathrm{H}$ Kelly, ${ }^{2} \mathrm{~J}$ Ngou, ${ }^{3} \mathrm{~B}$ Sawadogo, ${ }^{4} \mathrm{~B}$ Muzah, ${ }^{1} \mathrm{C}$ Gilham, ${ }^{2} \mathrm{~N}$ Nagot, ${ }^{3} \mathrm{~N}$ Meda, ${ }^{4} \mathrm{~S}$ Delany, ${ }^{2} \mathrm{M}$ Segondy, ${ }^{1 P}$ Mayaud. 'London School of Hygiene and Tropical Medicine, London, UK; ${ }^{2}$ University of Montpellier 1 \& INSERM U1058, Montpellier, France; ${ }^{3}$ Centre de Recherche Internationale sur la Sante, Ouagadougou, Burkina Faso; ${ }^{4}$ Wits Reproductive Health and HIV Institute, Johannesburg, South Africa

Background To assess the prevalence, genotype distribution and risk factors for high-risk HPV among HIV-positive African women, and associations with cervical histological lesions.

Methods The HARP study enrolled HIV-positive women aged 25-50 in Burkina Faso (BF) and South Africa (SA). A stratified sampling strategy was used, with $2 / 3$ of women on ART. Cervical HPV genotyping was performed using InnoLipa. Four-quadrant cervical biopsies were obtained among women with abnormalities detected by at least one test or by colposcopy.

Results 628 and 624 women were enrolled in BF and SA, respectively. The distribution of CD4+ count (cells $/ \mu \mathrm{L})$ was similar in both sites: $68 \%$ with CD $4+\geq 350$ and $10 \%$ with CD $4+<200$. Prevalence of HR-HPV genotypes was $62 \%$ among women in BF and $78 \%$ in SA, and, overall, $67 \%, 73 \%$ and $84 \%$ among women with CD4 $+\geq 350,200-349$ and $<200$, respectively (Table). The 4 most common genotypes in BF were HPV52 (20\%), HPV51 (12\%), HPV35 (9\%), HPV66 (8\%); and in SA, HPV52 (24\%), HPV16 (15\%), HPV51 $(14 \%)$ and HPV35 (14\%). Multiple types were observed in $41 \%$ and $55 \%$ of HR-HPV-positive women in BF and SA, respectively; and increased with decreasing CD4 count $(46 \%, 52 \%$ and $63 \%$, respectively, P-trend $=0.004)$. HPV types 58,33 and 16 were most strongly associated with CIN2 $+(\mathrm{OR}=5.06, \mathrm{OR}=4.62, \mathrm{OR}=4.02)$ and types 16,35 and 58 were most strongly associated with CIN3+ $(\mathrm{OR}=4.59, \mathrm{OR}=3.36, \mathrm{OR}=2.96)$. Decreasing $\mathrm{CD} 4+$ count and younger age were associated with higher HR-HPV prevalence in both countries (Table). Multiple sex partners, smoking and lower income were also significantly associated with HR-HPV in SA.

Conclusions HR-HPV prevalence is high among HIV-positive women with genotype distribution similar in both countries. HRHPV prevalence is associated with young age and lower CD4+ count. Whilst HPV52 is the most prevalent type, HPV16 is most strongly associated with increasing lesion severity.
Abstract P3.223 Table 1 Table. Association of HR genotypes with site, CD4+ count and age in Burkina Faso and South Africa

\begin{tabular}{lll}
\hline & \multicolumn{2}{l}{ High risk HPV genotypes } \\
\hline & $\mathrm{n} / \mathrm{N}(\%)$ & $\mathrm{OR}(95 \% \mathrm{Cl})$ \\
Site & & $\mathrm{P}<0.001$ \\
Burkina Faso & $285 / 463(62 \%)$ & 1 \\
South Africa & $385 / 492(78 \%)$ & $2.24(1.69-2.99)$ \\
CD4+ count (cells/ $\mathrm{LL})$ & & $\mathrm{P}$-trend $=0.001$ \\
$<200$ & $76 / 90(84 \%)$ & 1 \\
$200-349$ & $151 / 206(73 \%)$ & $0.51(0.26-0.97)$ \\
$>=350$ & $442 / 658(67 \%)$ & $0.38(0.21-0.69)$ \\
Age group & & $\mathrm{P}$-trend $=0.002$ \\
$25-29$ & $140 / 178(79 \%)$ & 1 \\
$30-34$ & $184 / 254(72 \%)$ & $0.71(0.45-1.12)$ \\
$35-39$ & $158 / 237(67 \%)$ & $0.54(0.35-0.85)$ \\
$40-49$ & $188 / 286(66 \%)$ & $0.52(0.34-0.80)$ \\
\hline
\end{tabular}

\section{P3.224 EFFECT OF HERPES SIMPLEX VIRUS TYPE 2 (HSV-2) INFECTION ON PROGRESSION OF HIV INFECTION AMONG FEMALE SEX WORKERS IN BURKINA FASO}

doi:10.1136/sextrans-2013-051184.0681

II T Traore, ${ }^{2}$ Konate, ${ }^{1,2} \mathrm{~N}$ Meda, ${ }^{2} \mathrm{~W}$ Bazie, ${ }^{1} \mathrm{M}$ N Hema, ${ }^{2} \mathrm{~A}$ Kabore, ${ }^{2} \mathrm{D}$ Kania, ${ }^{3 \mathrm{P}}$ Mayaud, ${ }^{4,5} \mathrm{P}$ Van De Perre, ${ }^{4,5} \mathrm{~N}$ Nagot. 'University of Ouagadougou, Ouagadougou, Burkina Faso; ${ }^{2}$ Centre Muraz, Bobo-Dioulasso, Burkina Faso; ${ }^{3}$ London School of Hygiene and Tropical Medicine, London, UK; ${ }^{4}$ University of Montpellier, Montpellier, France; ${ }^{5}$ Unité INSERM U1058, Montpellier, France

Background The effect of HSV-2 on the natural history of HIV-1 remains unclear. Although trials have shown a modest but significant impact of HSV-2 suppression on HIV-1 disease progression, the sub-optimal antiviral efficacy of aciclovir and its potential antiretroviral effect have limited our ability to measure the true effect of HSV-2 on HIV-1 disease progression. This study aimed to assess the effect of untreated HSV-2 infection on the time to ART.

Methods From December 2003 to February 2012, HIV-1 infected female sex workers were enrolled in a prospective open cohort in Burkina Faso. At each 3-month follow-up visits, CD4 count and HIV-1 plasma viral load were done. Participants were offered care including ART and psychological support. Participants not on ART and having at least $350 \mathrm{CD} 4$ cells/ $\mu \mathrm{l}$ at enrolment (the current CD4 count threshold for ART initiation) were included in this analysis, which was censored at 36 months of follow-up when the assumption of proportional hazard was no longer met.

Results Overall, 164 co-infected women and 20 HIV-1 monoinfected women were enrolled in this study. At enrollment, the only difference between the two groups was a younger age of HIV-1 mono-infected women (median age 24 versus 31 years, $p<0.001$ ). In linear mixed models, the age-adjusted mean CD4 count at baseline (intercept) was significantly lower among HSV-2 positive women ( -211 cell $/ \mu \mathrm{L}, \mathrm{p}<0.001)$, but no difference in baseline CD4-adjusted plasma viral load was observed. During follow-up, 3 out 20 HIV-1 mono-infected women initiated ART versus 52 out of $164 \mathrm{HSV}-2$ co-infected women. After adjustment for baseline CD4 count and age, HSV-2 infected women were still much more likely to initiate ART over 36 months (HR = 4.6, CI 95\%: 1.04-20.5, p = 0.04).

Conclusion HIV-1 disease progression, as assessed by time to ART eligibility, was much accelerated for women co-infected with HSV-2.

\section{P3.225 HSV-2 SEROPREVALENCE AMONG CURRENT INJECTION DRUG USERS IN ESTONIA}

doi:10.1136/sextrans-2013-051184.0682 